\title{
Gallic Acid-loaded Cellulose Acetate Electrospun Nanofibers: Thermal Properties, Mechanical Properties, and Drug Release Behavior
}

\author{
Manisara Phiriyawirut ${ }^{1 *}$, Thawatchai Phaechamud ${ }^{2}$ \\ ${ }^{1}$ Department of Tool and Materials Engineering, Faculty of Engineering, King Mongkut’s \\ University of Technology Thonburi, Bangkok, Thailand \\ ${ }^{2}$ Department of Pharmaceutical Technology, Faculty of Pharmacy, Silpakorn University, Nakhon Pathom, Thailand \\ Email: *manisara.pee@kmutt.ac.th
}

Received December 28, 2011; revised January 28, 2012; accepted February 7, 2012

\begin{abstract}
The gallic acid-loaded electrospun cellulose acetate fibers were successfully prepared. The fiber containing $2.5 \%$ gallic acid was smooth surface but observed drug flake on the surface of the fiber when increasing drug content. The thermal properties, mechanical properties and drug release behavior of the fibers were investigated comparing to the corresponding films.
\end{abstract}

Keywords: Electrospinning; Gallic Acid; Cellulose Acetate; Melting; Drug Release

\section{Introduction}

Polymer fibers are used in a wide variety of applications ranging from textiles to composite reinforcements. Traditional methods for obtaining polymer fibers include melt spinning [1], wet spinning and gel spinning [2]. Recently, there has been increasing interest in another method of fiber production, i.e., electrostatic spinning or electrospinning, which can produce fibers that are submicrometers down to nanometers in diameter.

The electrospinning technique is a simple and versatile method which utilizes a high voltage source to inject charge of a certain polarity into a polymer solution or melt and then accelerated toward a collector of opposite polarity. As the electrostatic attraction between the oppositely charged liquid and collector and the electrostatic repulsions between like charges in the liquid become stronger the leading edge of the solution changes from a rounded meniscus to a cone (the Taylor cone) [3]. A fiber jet is eventually ejected from the Taylor cone as the electric field strength exceeds the surface tension of the liquid. The fiber jet travels through the atmosphere allowing the solvent to evaporate, thus leading to the deposition of solid polymer fibers on the collector. The capacity to easily produce materials at this biological size scale has created a renewed interest in electrospinning for applications in drug delivery. The fibers exhibit several interesting characteristics, for example, a high surface area to mass or volume ratio, high density of pores in sub-micrometer length scale, vast possibilities for surface function- alization, etc. [4,5]. These unique properties have triggered a broad range of potential applications, including nanocomposites [6], scaffolds for tissue engineering [7], sensors [8], protective clothing and filtration membranes [9], magneto-responsive fibers [10], superhydrophobic membranes [11], and especially drug delivery [12].

Cellulose acetate (CA) is one of the most important organic esters derived from cellulose. It is widely used as fibers, plastics applications and membranes in many industrial applications. Cellulose acetate is manufactured by reacting cellulose with acetic anhydride using sulfuric acid as a catalyst. Liu and Hsieh [13] reported the preparation of ultrafine CA fiber mats as well as regenerated cellulose membranes by electrospinning. They found that the most suitable solvent system for preparing the CA solutions for electrospinning was $2: 1 \mathrm{v} / \mathrm{v}$ acetone/dimethylacetamide (DMAc), as this mixture allowed the resulting CA solutions (i.e., 12.5 - $20 \mathrm{wt} \%$ ) to be electrospun into fibers with average diameters ranging between 100 $\mathrm{nm}$ and $1 \mathrm{~mm}$.

The applications of electrospun CA fiber mat as carriers for topical/transdermal delivery of drugs have been reported [12,14] and [15]. Taepaiboon et al. [12] developed electrospun CA fiber mats as carriers for topical/transdermal delivery of vitamin A acid (Retin-A) and vitamin E (Vit-E). Moreover, Suwantong et al. [15] studied the use of electrospun CA fiber mats as carriers of curcumin, a herbal compound found in the plant Curcuma longa $L$. 
Among various drug, gallic acid (3,4,5-trihydroxybenzoic acid) is a natural phenolic compounds mostly found in gallnuts, sumac, witch hazel, grapes, oak bark, and green tea. It has several biological activities including antioxidant, antityrosinase, antimicrobial, anti-inflammatory and anticancer activities [16-18]. Due to its promising antioxidant activity, gallic acid is added in various skincare products in the form of natural herbal extracts. Gallic acid is also used as a standard substance in many antioxidant assays. Gallic acid has been shown to possess scavenging activities against several radicals, for example: superoxide anion, hydroxyl radicals, singlet oxygen or peroxyl radicals, and to protect cells from damage induced by UV-B or ionizing irradiation [19]. Additionally, gallic acid showed an antibacterial property against Escherichia coli, Staphylococcus aureus, Pseudomonas aeruginosa and especially Klebsiella pneumoniae [20]. From above mentioned this substance has many biological activities that has been potentially developed as active compound for skin or transdermal delivery system. However, the main limitation of gallic acid is its poor water solubility $(11.5 \mathrm{mg} / \mathrm{mL})$ [21,22]. In order to overcome this problem, electrospun fiber mats was use as a drug delivery carrier of gallic acid due to their high surface area should improve the solubility or releasing of gallic acid.

The aim of this research was to develop mats of electrospun CA nanofibers as carriers for delivery of gallic acid to the skin. In the present contribution, gallic acid was loaded into a CA solution which was later fabricated into ultrafine fibers by electrospinning. The gallic acidloaded electrospun CA fiber mats were assessed for their potential use as carriers for topical or transdermal delivery of gallic acid. Morphology, thermal and tensile properties of both the neat and the gallic acid-loaded electrospun CA fiber mats were investigated. The gallic acid release behavior of the gallic acid-loaded electros-pun CA fiber mats was also investigated. Comparisons were made against the corresponding solvent-cast films.

\section{Experimental}

\subsection{Materials}

Cellulose acetate (CA; white powder; $\mathrm{M}_{\mathrm{w}}=30,000 \mathrm{Da}$; acetyl content $=39.7 \mathrm{wt} \%$; degree of acetyl substitution 2.4) was purchased from Sigma-Aldrich (Switzerland). Acetone and $\mathrm{N}, \mathrm{N}$-dimethylacetamide (DMAc), were purchased from Labscan (Asia), Thailand. Gallic acid was purchased from Fluka.

\subsection{Fiber Mat Preparation by Electrospinning}

An initial weighed amount of CA powder was dissolved in $2: 1 \mathrm{v} / \mathrm{v}$ acetone/DMAc to obtain a $17 \% \mathrm{w} / \mathrm{w}$ CA solution as mentioned in the literature [23]. Gallic acid- loaded CA solutions were prepared by dissolving the same amount of CA powder and gallic acid in the amount of 2.5 - $10 \mathrm{wt} \%$ based on the weight of CA powder, respectively, in the acetone/DMAc mixture.

Electrospinning of the as-prepared solutions was carried out by connecting the emitting electrode of positive polarity from a Gamma High-Voltage Research ES30PN/ M692 high voltage DC power supply to the solutions contained in a standard 50-ml syringe, the open end of which was attached to a blunt gauge-20 stainless steel needle $(\mathrm{OD}=1.2 \mathrm{~mm})$, used as the nozzle, and the grounding electrode to a home-made rotating metal drum $(\mathrm{OD}=12 \mathrm{~cm})$ was covered with aluminum foil to used as the fiber collection device. Electrical potential of $12 \mathrm{kV}$ was applied across a fixed distance of $12.5 \mathrm{~cm}$ between the tip of the nozzle and the outer surface of the drum. The feed rate of the solutions was controlled at $0.1 \mathrm{ml} / \mathrm{h}$ by means of a Kd Scientific syringe pump. Electrospinning was carried out in room conditions.

In order to compared the mechanical properties and drug release behavior of as-prepared electrospun fiber mat, gallic acid-loaded films were prepared by casting technique.

\subsection{Characterizations and Testing}

Prior to electrospinning, the as-prepared solutions were measured for their viscosity and conductivity using a viscometer (VISCO STAR Plus) and a conductivity meter (Oakton CON 6/TDS 6 Conductivity/TDS meters), respectively. The measurements were carried out at $25^{\circ} \mathrm{C}$ and average values for each solution were calculated from at least three measurements.

Morphological appearance of the neat and the gallic acid-loaded as-spun CA fiber mats were observed by a JEOL JSM-6380LV scanning electron microscope (SEM). The fiber mats samples were sputtered with a thin layer of gold prior to SEM observation. Diameters of the individual fibers in the as-spun fiber mats were measured directly from the SEM images using a SemAphore 4.0 software.

Thermal properties of the neat and the gallic acidloaded electrospun CA fiber mats were analyzed by differential scanning calorimetry (DSC) and observed the melting behavior by hot stage microscope. DSC thermograms were recorded on a Perkin Elmer Pyris Sapphine DSC analyzer, standard $115 \mathrm{~V}$ class A at a heating rate of $10^{\circ} \mathrm{C} \cdot \mathrm{min}^{-1}$ under nitrogen atmosphere. Samples of around $5 \mathrm{mg}-10 \mathrm{mg}$ were used for the DSC measurements. Melting behavior were observed by hot stage microscope on hot stage, Mettler-Toledo GmbH Analytical, FP90 Central Processor at a heating rate of $15^{\circ} \mathrm{C} \cdot \mathrm{min}^{-1}$. Recording the change of sample was performed every one minute.

The mechanical integrity in terms of the tensile strength 
and the strain at maximum of both the neat and the gallic acid-loaded as-spun CA fiber mats and as-cast CA films were investigated using a Texture analyzer model Stable Micro System TA.XT. plus at room conditions. Each specimen was cut into a rectangular shape $(10 \times 90 \mathrm{~mm})$. The crosshead speed and the gauge length were $20 \mathrm{~mm} /$ min and $50 \mathrm{~mm}$, respectively. The results were reported as average values $(n=10)$.

The percentage of swelling and the percentage of weight loss of both the neat and the gallic acid-loaded as-spun CA fiber mats were measured in a phosphate buffer solution at temperature of $25^{\circ} \mathrm{C}$ for $24 \mathrm{~h}$ according to the Equation (1) and Equation (2), respectively:

$$
\begin{gathered}
\text { Swelling }(\%)=\frac{M-M_{i}}{M_{i}} \\
\text { Weight loss }(\%)=\frac{M_{i}-M_{d}}{M_{i}}
\end{gathered}
$$

where $M$ is the weight of each sample after submersion in the buffer solution for $24 \mathrm{~h}, M_{d}$ is the weight of the sample after submersion in the buffer solution for $24 \mathrm{~h}$ in its dry state, $M_{i}$ is the initial weight of the sample in its dry state.

Gallic acid release behavior was determined by monitoring the UV absorbance as a function of release time. The gallic acid-loaded as-spun CA fiber mats were first punched into phosphate buffer solution medium $\mathrm{pH}$ 6.8, the gallic acid release studies were carried out at room temperature. Samples of $5 \mathrm{~mL}$ were taken from the buffer solution between 0 - 168 hours. The amount of gallic acid present in the release buffer was determined by a UV-vis spectrophotometer UV-2101 PC (Shimadsu, Japan) at the wavelength of $259 \mathrm{~nm}$. The results were presented in term of cumulative release as a function of release time based on Equation (3):

$$
\text { Cumulative amount of release }(\%)=\frac{M_{t}}{M_{100}} \times 100
$$

where $M_{t}$ was the amount of gallic acid released at time t, the amount of gallic acid in gallic acid-loaded as-spun CA fiber mats was regarded as $M_{100}$. Five samples were tested for each gallic acid-loaded as-spun CA fiber mats.

\section{Results and Discussion}

\subsection{Electrospinning}

Due to limited soluble ability of gallic acid in CA solution, the CA solution containing 2.5\% - 5\% gallic acid was yellowish clear solution but when increasing the content of gallic acid to $7.5 \%$ - $10 \%$ the colloidal solution were achieved and the color of the solution was turn to yellow with more gallic acid content. Prior to electrospinning, both the neat and the gallic acid-loaded CA solutions were measured for their viscosity and the conductivity, and the results are summarized in Table 1.

The presence of gallic acid in the base CA solution was responsible for the observed increase in the viscosity values but the solution conductivity was found to slightly decrease with increasing the gallic acid content. Electrospinning of these solutions was carried out at a fixed electric field of $12 \mathrm{kV} / 12.5 \mathrm{~cm}$. Selected SEM images of the electrospun fibers from these solutions are shown in Figure 1.

Table 1. Shear viscosity and electrical conductivity of neat and gallic acid-containing CA solutions $(n=10)$ as well as diameters and fiber content of the individual fibers within the resulting electrospun fiber mats.

\begin{tabular}{ccccc}
\hline $\begin{array}{c}\text { Gallic acid } \\
\text { content } \\
(\% \mathrm{w} / \mathrm{w})\end{array}$ & $\begin{array}{c}\text { Viscosity } \\
(\mathrm{cP})\end{array}$ & $\begin{array}{c}\text { Electrical } \\
\text { conductivity } \\
\left(\mathrm{mS} \mathrm{cm}^{-1}\right)\end{array}$ & $\begin{array}{c}\text { Fiber } \\
\text { Diameters } \\
(\mathrm{nm})\end{array}$ & $\begin{array}{c}\text { Fiber } \\
\text { content } \\
(/ 100 \mathrm{um})\end{array}$ \\
\hline 0 & 498.5 & 4.45 & $539 \pm 213$ & $17 \pm 2$ \\
2.5 & 542.2 & 4.32 & $701 \pm 162$ & $23 \pm 1$ \\
5 & 686.2 & 4.28 & $716 \pm 160$ & $18 \pm 3$ \\
7.5 & 832.1 & 4.13 & $935 \pm 142$ & $12 \pm 2$ \\
10 & 973.2 & 4.04 & $1057 \pm 298$ & $11 \pm 3$ \\
\hline
\end{tabular}

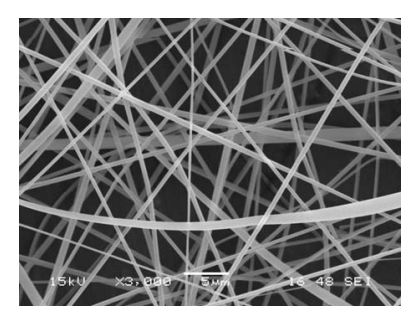

(a)

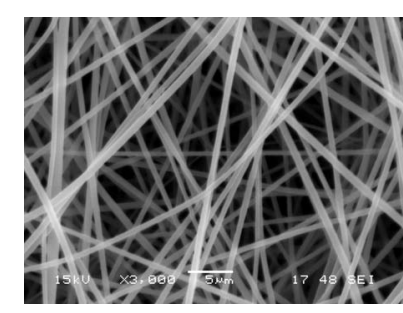

(c)

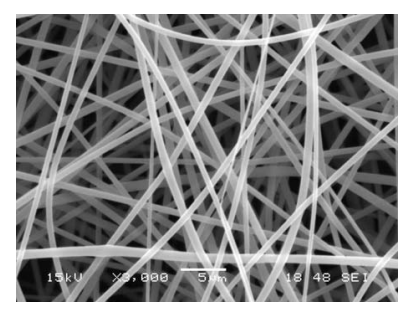

(b)

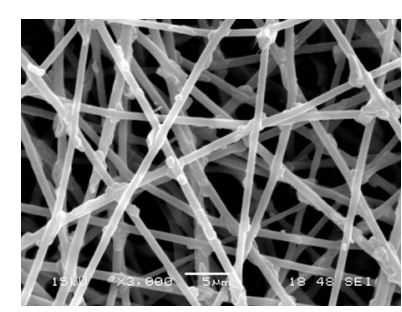

(d)

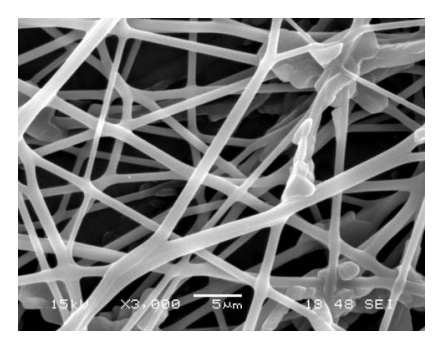

(e)

Figure 1. Scanning electron micrographs of neat and gallic acid-loaded electrospun CA fiber mats at various gallic acid contents for (a) $0 \%$; (b) $2.5 \%$; (c) $5 \%$; (d) $7.5 \%$ and (e) $10 \%$. Note: applied electric field $=12 \mathrm{kV} / 12.5 \mathrm{~cm}$. 
Clearly, cross-sectionally round fibers were obtained. The diameters of the neat CA fibers were $\sim 539 \pm 213 \mathrm{~nm}$, while those of the gallic acid-loaded CA fibers ranged between $\sim 701 \pm 162$ and $1057 \pm 298 \mathrm{~nm}$ with dependency on the initial amount of the as-loaded gallic acid (see Table 1). The neat CA fiber was a smallest size when compared to the gallic acid-loaded CA fiber. This is because of the lowest solution viscosity and highest electrical conductivity which accelerate the stretching of the polymer during electrospinning. An adding the gallic acid to CA solution was affected the viscosity of the CA solution and resulted in increasing the fiber diameter. Normally, the solution viscosity increased with increasing solution concentration according to a power-law relationship and an increase in the solution viscosity should result in the formation of fibers of larger diameters [24, 25].

Interestingly, the aggregation of gallic acid was observed on the surface of these fibers especially with gallic acid content of $7.5 \%$ - $10 \%$, implying that the as-loaded gallic acid was not perfectly incorporated well within the fibers. Gallic acid is poor water solubility [22]. Its solubility in the electrospinning solution was limited. During the rapid evaporation of the solvent, phase separation took place quickly between the drug and CA. Therefore, quite a portion of the drug aggregate came to the fiber surface.

\subsection{Thermal Properties and Melting Behavior}

The thermal properties of gallic acid, the neat and the gallic acid-loaded electrospun CA fiber mats were investigated by DSC and hot stage microscope. Figure 2 shows DSC thermograph for the gallic acid and the neat and the gallic acid-loaded electrospun CA fiber mats. Within the temperature range investigated, gallic acid showed the large board exothermic, the loss of moisture coupled, and the melting transition, with the peak temperatures being observed at $62^{\circ} \mathrm{C}$ and $267^{\circ} \mathrm{C}$, respectively.

The neat electrospun CA fiber mat exhibited only endothermic thermal transitions, $50^{\circ} \mathrm{C}-110^{\circ} \mathrm{C}$, corresponding to the loss of moisture coupled with a glass transition but absent the melting peak. The reported glass transition temperature of CA was occurred over a wide temperature range depending on the degree of acetyl substitution (DS) of the polymer, [26] while the reported melting of CA (degree of polymerization $\approx 500-600$; $\mathrm{DS} \approx 2.4$ ) in the form of solvent-cast film was occurred over a wide temperature range with the peak temperature being observed at $224^{\circ} \mathrm{C}$ [27].

All of the gallic acid-loaded electrospun CA fiber mats exhibited a loss of moisture coupled with a glass transition over about the same temperature range as that of the neat electrospun CA fiber mat. Moreover, it was found that the area under the endothermic peak increased with

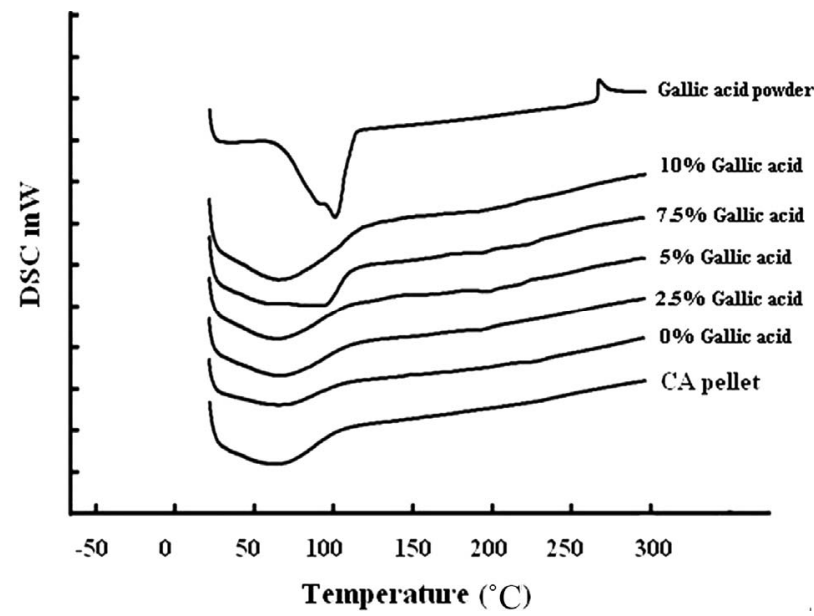

Figure 2. DSC thermogram of gallic acid powder, CA pellet, neat and gallic acid-loaded electrospun CA fiber mats.

increasing gallic acid content. It is postulated that an incorporation of the gallic acid into the CA fiber mats was improved the moisture absorption of the electrospun CA fiber mats. However, the melting peak which related to the melting of gallic acid was absent from the corresponding DSC thermograms for the gallic acid-loaded electrospun CA fiber mats. Since the very low concentration of gallic acid in electrospun CA fiber mats, it is possible that it could not be detected by DSC technique.

In order to study the melting behavior of gallic acidloaded electrospun CA fiber mats, hot stage microscope was used and reported in Table 2. It was found that the melting temperature of the neat electrospun CA mat started at $212^{\circ} \mathrm{C}$. Evidently, onset melting temperature of gallic acid-loaded electrospun CA fiber mats was found to decrease with increasing gallic acid content.

\subsection{Mechanical Properties}

The mechanical properties in terms of the tensile strength and the elongation at break of both the neat and the gallic acid-loaded electrospun CA fiber mats were investigated and the results are summarized in Table 3.

The initial addition of gallic acid (i.e., at $2.5 \% \mathrm{w} / \mathrm{w}$ ) in the CA solution caused both the tensile strength and elongation at break of the gallic acid-loaded electrospun CA fiber mats to increase from that of the neat fiber mats. With further increase in the gallic acid content between $2.5 \%$ and $7.5 \% \mathrm{w} / \mathrm{w}$ in the CA solution, the tensile strength of the gallic acid-loaded electrospun CA fiber mats increased monotonically with increase in the gallic acid content to reach maximum values at the gallic acid content of $7.5 \% \mathrm{w} / \mathrm{w}$. Further increase in the gallic acid content to $10 \% \mathrm{w} / \mathrm{w}$, a decrease in the tensile strength of the gallic acid-loaded electrospun CA fiber mats was observed. On the other hand, the elongation at break of the electrospun CA fiber mats that were fabricated from 
Table 2. Melting behavior of neat and gallic acid-loaded electrospun CA fiber mats.

\begin{tabular}{|c|c|c|c|c|c|}
\hline \multirow{2}{*}{$\begin{array}{c}\text { Gallic acid } \\
\text { content }(\% \mathrm{w} / \mathrm{w})\end{array}$} & \multirow{2}{*}{$\begin{array}{c}\text { Before melting } \\
\text { Appearance }\end{array}$} & \multicolumn{2}{|c|}{ Onset melting } & \multicolumn{2}{|c|}{ After melting } \\
\hline & & $\begin{array}{c}\mathrm{T} \\
\left({ }^{\circ} \mathrm{C}\right)\end{array}$ & Appearance & $\begin{array}{c}\mathrm{T} \\
\left({ }^{\circ} \mathrm{C}\right)\end{array}$ & Appearance \\
\hline 0 & & 212 & & 248 & \\
\hline 2.5 & & 191 & & 227 & \\
\hline 5 & & 189 & & 225 & \\
\hline 7.5 & & 178 & & 220 & \\
\hline 10 & & 180 & & 222 & \\
\hline
\end{tabular}

Table 3. Mechanical properties of neat and gallic acid-loaded electrospun CA fiber mats.

\begin{tabular}{ccccc}
\hline \multirow{2}{*}{ Gallic acid content $(\% \mathrm{w} / \mathrm{w})$} & \multicolumn{2}{c}{ Tensile strength (MPa) } & \multicolumn{2}{c}{ Flongation at break (\%) } \\
\cline { 2 - 5 } & Fiber mat & Film & $22.10 \pm 2.99$ & $1.827 \pm 0.44$ \\
\hline 0 & $1.08 \pm 0.31$ & $26.62 \pm 4.98$ & $28.04 \pm 2.77$ & $3.16 \pm 0.28$ \\
5.5 & $1.13 \pm 0.25$ & $23.72 \pm 4.94$ & $15.21 \pm 8.93$ & $2.73 \pm 0.68$ \\
7.5 & $1.36 \pm 0.15$ & $14.38 \pm 1.63$ & $13.92 \pm 1.05$ & $1.38 \pm 0.58$ \\
10 & $1.83 \pm 0.27$ & $11.28 \pm 1.42$ & $10.88 \pm 12.02$ & $1.81 \pm 0.69$ \\
\hline
\end{tabular}

the CA solutions containing 5\% - 10\% w/w gallic acid was significant decrease monotonically with increase in the gallic acid content.

On the other hand, the tensile strength for all the as- cast films was in the range of 10.45 - $26.62 \mathrm{MPa}$, which decreased with increasing gallic acid. The much greater tensile strength of the as-cast films in comparison with that of the electrospun fiber mats was also reflected in 
the observed lower elongation at break of the films in comparison with that of the electrospun fiber mats. Specifically, the elongation at break for all the as-cast films was in the range of $1.38 \%-2.73 \%$. This result was also agreed to previous reports $[12,15]$.

\subsection{Swelling and Weight Loss Behavior}

The percentage of swelling and the percentage of weight loss of the gallic acid-loaded electrospun CA fiber mats after submersion in phosphate buffer $\mathrm{pH} 6.8$ at $25^{\circ} \mathrm{C}$ for $24 \mathrm{~h}$ were characterized and the results are shown in Figure 3.

The percentage of swelling of electrospun CA fiber mats was $2527 \%$. In comparison with that of the neat materials, the swelling ability of the gallic acid-loaded electrospun CA fiber mats prepared from the CA solutions containing 2.5\% w/w gallic acid was higher (i.e., 3124\%), while that of gallic acid-loaded electrospun CA fiber mats prepared from the solutions containing 5, 7.5 and $10 \%$ $\mathrm{w} / \mathrm{w}$ gallic acid was lower. An increase in percentage

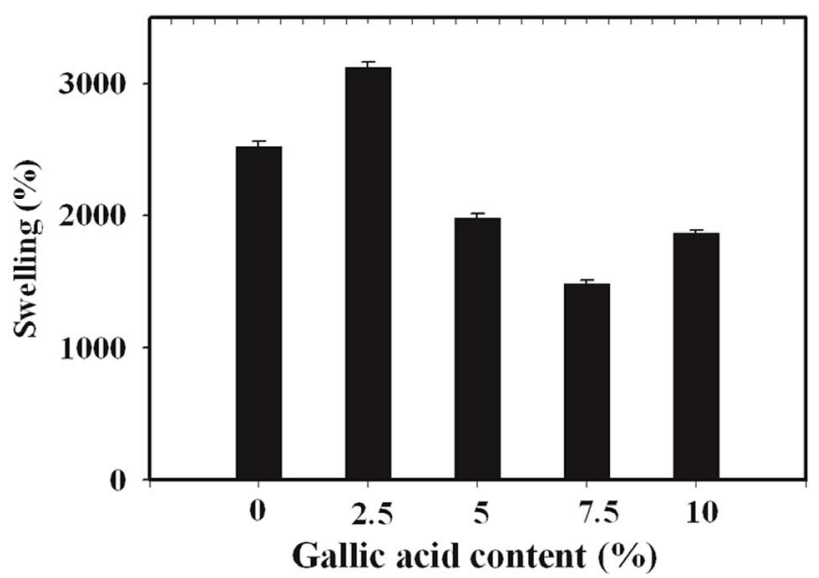

(a)

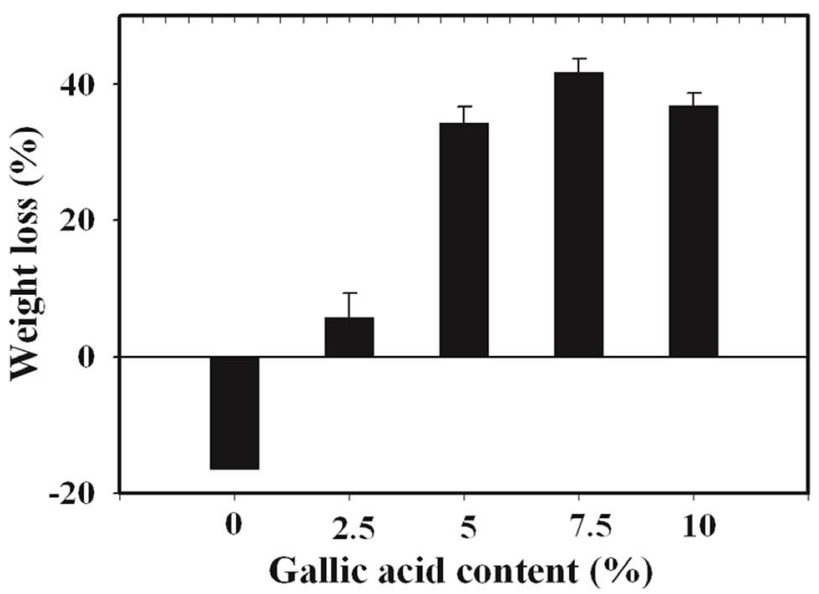

(b)

Figure 3. (a) Percentage of swelling and (b) percentage of weight loss in phosphate buffer at room temperature for neat and the gallic acid-loaded electrospun CA fiber mats. of swelling of $2.5 \% \mathrm{w} / \mathrm{w}$ gallic acid-loaded electrospun CA fiber mats was due to the good incorporation of gallic acid into the electrospun CA fiber mats which increased the water absorption ability of the electrospun fiber mats. In the contrast way, a decrease in percentage of swelling of higher loading content of gallic acid-loaded electrospun CA fiber mats was due to the aggregation of gallic acid on the surface of the electrospun CA fiber mats which easy to dissolve in buffer media. The water solubility of gallic acid is $11.5 \mathrm{mg} / \mathrm{mL}$ [22]. This phenomenon is results in lowering the weight of sample. However, due to the high water absorption ability of the CA molecule so these gallic acid-loaded electrospun CA fiber mats were still swelling in buffer medium but lower when compared to the neat materials.

Evidently, the percentage of weight loss for the neat electrospun CA fiber mats after submersion in phosphate buffer for $24 \mathrm{~h}$ was in the range of $-18 \%$. Since CA is soluble in glacial acetic acid [26], it should be less soluble in this phosphate buffer solution. Moreover, due perhaps to the high ability to form the strongly chemical bonding to water molecule of the neat fiber mats, the percentage of weight loss for both types of neat electrospun CA fiber was then low.

In comparison with those of the neat fiber mats, such the percentage of weight loss values of the gallic acidloaded electrospun CA fiber mats was increased with increasing gallic acid loading content. These corresponded to the percentage of swelling which as a result of dissolution of gallic acid aggregate on the surface of the electrospun fiber mats.

\subsection{Gallic Acid Release Behavior}

The release characteristics of the gallic acid from the gallic acid-loaded electrospun CA fiber mats and as-cast CA films were carried out by the total immersion method. The experiments were carried out in the phosphate buffer medium at the room temperature. The cumulative amount of the gallic acids released (reported as the percentage of the actual amount of gallic acids present in the drugloaded samples) from the drug-loaded samples is showed in Figure 4.

Gallic acid released from the drug-loaded as-cast film exhibited a burst release, while that from the electrospun CA fiber mats ones did not. Specifically, gallic acid released from the as-cast film showed the burst release during the first $20 \mathrm{~min}$ and reached a plateau at the maximum release of $15 \%$ with further increase in the immersion time for $2.5 \%$ gallic acid content as-cast film and $20 \%$ for $10 \%$ gallic acid content as-cast film. On the other hand, the drug-loaded electrospun fiber mat showed a gradual increase in the cumulative release of gallic acid over the $100 \mathrm{~h}$ testing period. At $100 \mathrm{~h}$, the maximum amount of gallic acid released from the electrospun fiber 


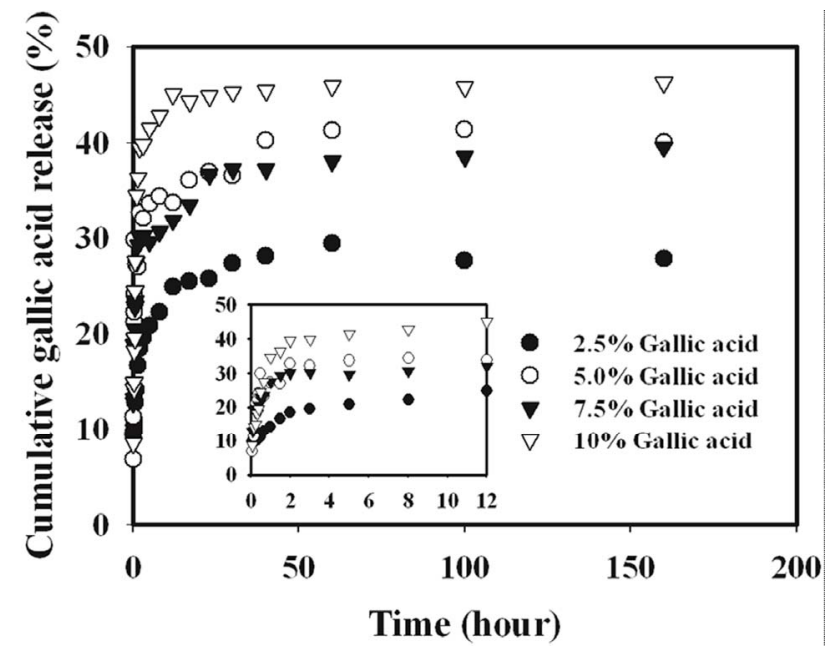

(a)

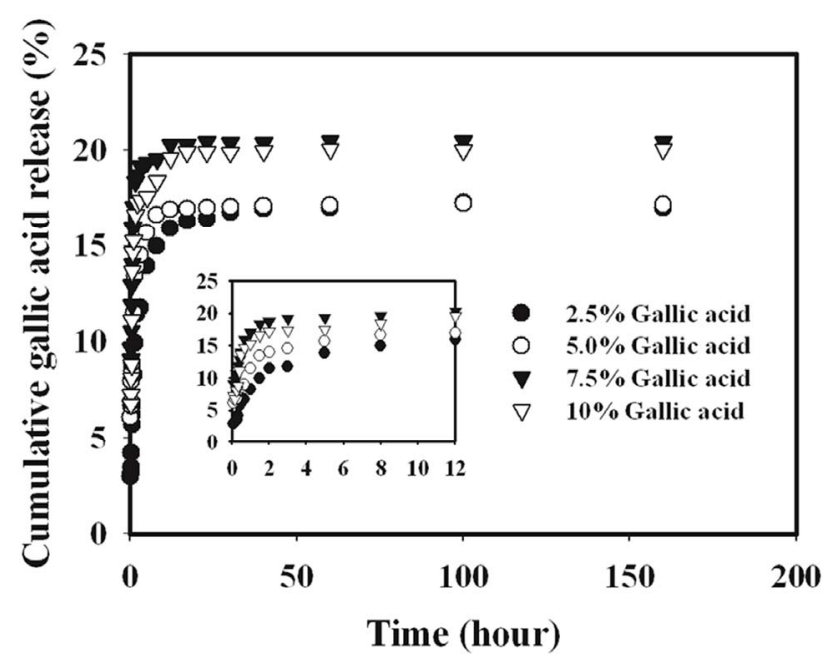

(b)

Figure 4. Cumulative gallic acid release profile in phosphate buffer medium pH 6.8 at room temperature from (a) electrospun CA fiber mats and (b) as-cast films.

mat samples was $45 \%$. The $2.5 \%$ gallic acid-loaded CA fiber mats specimen was released into the medium in range of $45 \%$ and the time for constant release of gallic acid was longer than that of other gallic acid content loaded CA fiber mats.

Interestingly, it was found that the release from $7.5 \%$ $10 \% \mathrm{w} / \mathrm{w}$ gallic acid loaded electrospun CA fiber mats were rather rapid in the initial stage of release profile when compared to that of the lower gallic acid loaded fiber mats, because at this loading content, the gallic acid was located on the surface of electrospun fiber under the driving of electric force in the process of electrospinning due to the limitation of gallic acid solubility in CA solution, as seen in SEM result. Thus, the drugs located on the surface of electrospun fibers could dissolve into buffer solution directly.

It is apparent that the amounts of gallic acid released from the drug-loaded as-cast CA films were significantly lower than those released from the corresponding electrospun fiber mats. The fact that the amounts of gallic acid released from the drug-loaded electrospun CA fiber mats were greater than that from the corresponding as-cast films could be due to the highly porous structure of the electrospun fiber mats that provided much greater surface area per unit volume or mass of the materials than the dense structure of the corresponding as-cast films [15].

\section{Conclusions}

Gallic acid-loaded electrospun cellulose acetate (CA) fiber mats were prepared by electrospinning technique under a fixed electric field of $12 \mathrm{kV} / 12.5 \mathrm{~cm}$. 17\% w/v CA solution in 2:1 v/v acetone/ $N, N$-dimethylacetamide was used as the base spinning solution, into which gallic acid in an amount of $2.5 \%-10 \% \mathrm{w} / \mathrm{w}$ (based on the weight of CA) was added to prepare the gallic acid spinning solutions. The electrospun fibers from these solutions were found to be cross-sectionally round with smooth surface for low gallic acid loading content but observed the aggregated gallic acid flake on the surface of fiber for high gallic acid loading content. The average diameters were ranging between 537 and $1057 \mathrm{~nm}$ which found to increase with increasing gallic acid loading content. The melting behavior of these electrospun fiber mats was depended on the gallic acid loading content which decreased with increasing gallic acid content. The mechanical properties in terms of the tensile strength and the percentage of elongation ate break of these electrospun fiber mats was evaluated in comparison with that of the corresponding as-cast films. It was observed that the as-cast films exhibited greater tensile strength than the electrospun fiber mats, while the percentage of elongation ate break of the electrospun fiber mats was about $10 \times$ as much as that of the as-cast films. The release characteristics of gallic acid from the gallic acid-loaded electrospun CA fiber mats and the corresponding as-cast films were tested in the phosphate buffer medium at room temperature, by the total immersion method. The loading content of gallic acid was significantly affected to the release behavior due to the different fiber morphology. In comparison with the amounts of gallic acid released from the drug-loaded electrospun CA fiber mats, those of the gallic acid released from the corresponding as-cast films were much lower.

\section{Acknowledgements}

The financial support was provided from the Research, Development and Engineering (RD\&E) Fund through National Nanotechnology Center (NANOTEC), National Science and Technology Department Agency (NSTDA), 
Thailand (Project No. NN-B-22-TT2-17-51-22). The authors would like to acknowledge the facility support from Department of Tool and Materials Engineering, Faculty of Engineering, King Mongkut's University of Technology Thonburi and Faculty of Pharmacy, Silpakorn University, Thailand. The authors would like to thank to Mr. Chaloemphon Chumpradid, Mr. Panuwat Tanasanskulwong, and Mr. Teeranan Wandee for their help.

\section{REFERENCES}

[1] J. Suh, J. E. Spruiell and S. A. Schwartz, "Melt Spinning and Drawing of 2-Methyl-1, 3-Propanediol-Substituted Poly(Ethylene Terephthalate)," Journal of Applied Polymer Science, Vol. 88, No. 11, 2003, pp. 2598-2606. doi:10.1002/app.11871

[2] P. J. Barham and A. Keller, "High-Strength Polyethylene Fibers from Solution and Gel Spinning," Journal of Materials Science, Vol. 20, No. 7, 1985, pp. 2281-2302. doi:10.1007/BF00556059

[3] D. H. Reneker and I. Chun, "Nanometre Diameter Fibres of Polymer, Produced by Electrospinning," Nanotechnology, Vol. 7, No. 3, 1996, pp. 216-223. doi:10.1088/0957-4484/7/3/009

[4] J. Doshi and D. H. Reneker, "Electrospinning Process and Applications of Electrospun Fibers,” Journal of Electrostatic, Vol. 35, No. 2-3, 1995, pp. 151-60. doi:10.1016/0304-3886(95)00041-8

[5] J. M. Deitzel, J. Kleinmeyer, D. Harris and N. C. B. Tan, "Electrospinning of Polymer Nanofibers with Specific Surface Chemistry,” Polymer, Vol. 42, No. 1, 2001, pp. 261-272. doi:10.1016/S0032-3861(00)00250-0

[6] D. Li, Y. Wang and Y. Xia, "Electrospinning of Polymeric and Ceramic Nanofibers as Uniaxially Aligned Arrays," Nano Letters, Vol. 3, No. 8, 2003, pp. 1167-1171. doi:10.1021/nl0344256

[7] H.-J. Jin, J. Chen, V. Karageorgious, G. H. Altman and D. L Kaplan, "Human Bone Marrow Stromal Cell Responses on Electrospun Silk Fibroin Mats,” Biomaterials, Vol. 25, No. 6, 2004, pp. 1039-1047. doi:10.1016/S0142-9612(03)00609-4

[8] X. Wang, Y. Kim, C. Drew, B. Ku, J. Kuma and L. A. Samuelson, "Electrostatic Assembly of Conjugated Polymer Thin Layers on Electrospun Nanofibrous Membranes for Biosensors," Nano Letters, Vol. 4, No. 2, 2004, pp. 331-334. doi:10.1021/nl034885z

[9] P. Gibson, H. Schreuder-Gibson and D. Rivin, “Transport Properties of Porous Membranes Based on Electrospun Nanofibers," Colloids and Surfaces A: Physicochemical and Engineering Aspects, Vol. 187-188, 2001, pp. 469481. doi:10.1016/S0927-7757(01)00616-1

[10] M. Wang, H. Singh, T. A. Hatton and G. C. Rutledge, "Field-Responsive Superparamagnetic Composite Nanofibers by Electrospinning," Polymer, Vol. 45, No. 16, 2004, pp. 5505-5514. doi:10.1016/j.polymer.2004.06.013

[11] M. L. Ma, R. M. Hill, J. L. Lowery, S. V. Fridrikh and G. C. Rutledge, "Electrospun Poly(Styrene-Block-Dimethylsiloxane) Block Copolymer Fibers Exhibiting Superhy- drophobicity,” Langmuir, Vol. 21, No. 12, 2005, pp. 55495554. doi:10.1021/la047064y

[12] P. Taepaiboon, U. Rungsardthong and P. Supaphol, "Vitamin-Loaded Electrospun Cellulose Acetate Nanofiber mats as Transdermal and Dermal Therapeutic Agents of Vitamin a Acid and Vitamin E," European Journal of Pharmaceutics and Biopharmaceutics, Vol. 67, No. 2, 2007, pp. 387-397. doi:10.1016/j.ejpb.2007.03.018

[13] H. Liu and Y. L. Hsieh, "Ultrafine Fibrous Cellulose Membranes from Electrospinning of Cellulose Acetate," Journal of Polymer Science Part B: Polymer Physics, Vol. 40, No. 18, 2002, pp. 2119-2129. doi:10.1002/polb.10261

[14] S. Tungprapa, I. Jangchud and P. Supaphol, "Release Characteristics of Four Model Drugs-Loaded Electrospun Cellulose Acetate Fiber Mats,” Polymer, Vol. 48, No. 17, 2007, pp. 5030-5041. doi:10.1016/j.polymer.2007.06.061

[15] O. Suwantong, P. Opanasopit, U. Ruktanonchai and P. Supaphol, "Electrospun Cellulose Acetate Fiber Mats Containing Curcumin and Release Characteristic of the Herbal Substance,” Polymer, Vol. 48, No. 26, 2007, pp. 75467557. doi:10.1016/j.polymer.2007.11.019

[16] R. Krogh, R. A. Yunes and A. D. Andricopulo, "Structure-Activity Relationships for the Analgesic Activity of Gallic Acid Derivatives,” II Farmaco, Vol. 55, No. 11-12, 2000, pp. 730-735. doi:10.1016/S0014-827X(00)00094-X

[17] L. Zhongbing, N. Guangjun, P. S. Belton, H. Tang and B. Zhao, "Structure-Activity Relationship Analysis of Antioxidant Ability and Neuroprotective Effect of Gallic Acid Derivatives," Neurochemistry International, Vol. 48, No. 4, 2006, pp. 263-274. doi:10.1016/j.neuint.2005.10.010

[18] S. Shahrzad, K. Aoyagi, A. Winter, A. Koyama and I. Bitsch, "Pharmacokinetics of Gallic Acid and Its Relative Bioavailability from Tea in Healthy Humans," Journal of Nutrition, Vol. 131, No. 4, 2001, pp. 1207-1210.

[19] T. Sawa, M. Nakao, T. Akaike, K. Ono and H. Maeda, "Alkylperoxyl Radical-Scavenging Activity of Various Flavonoids and Other Phenolic Compounds: Implications for the Anti-Tumor-Promoter Effect of Vegetables,” Journal of Agricultural and Food Chemistry, Vol. 47, No. 2, 1999, pp. 397-402. doi:10.1021/jf980765e

[20] V. M. J. Rodrguez, M. R. Alberto and M. M. C. de Nadra, "Antibacterial Effect of Phenolic Compounds from Different Wines,” Food Control, Vol. 18, No. 2, 2007, pp. 93-101. doi:10.1016/j.foodcont.2005.08.010

[21] M. J. O’Neil, A. Smith, P. E. Heckelman, J. R. Obenchain, J. A. R. Gallipeau and M. A. D’Arecca, “Gallic Acid,” In: M. J. O’Neil, A. Smith, P. E. Heckelman, J. R. Obenchain, J. A. R. Gallipeau and M. A. D’Arecca, Eds., The Merck index,13th Edition, Merck Research Laboratories, New Jersey, 2001, p. 261.

[22] C. M. Hansen and L. Just, "Prediction of Environmental Stress Cracking in Plastics with Hansen Solubility Parameters,” Industrial \& Engineering Chemistry Research, Vol. 40, No. 1, 2001, pp. 21-25. doi:10.1021/ie9904955

[23] P. Taepaiboon, U. Rungsardthong and P. Supaphol, “DrugLoaded Electrospun Mats of Poly(Vinyl Alcohol) Fibres and Their Release Characteristics of Four Model Drugs," Nanotechnology, Vol. 17, No. 9, 2006, pp. 2317-2329. doi:10.1088/0957-4484/17/9/041 
[24] C. Mit-Uppatham, M. Nithitanakul and P. Supaphol, "Ultrafine Electrospun Polyamide-6 Fibers: Effect of Solution Conditions on Morphology and Average Fiber Diameter," Macromolecular Chemistry and Physics, Vol. 205, No. 17, 2004, pp. 2327-2338.

doi:10.1002/macp.200400225

[25] C. Mit-uppatham, M. Nithitanakul and P. Supaphol, "Effects of Solution Concentration, Emitting Electrode Polarity, Solvent Type, and Salt Addition on Electrospun Polyamide-6 Fibers: A Preliminary Report," Macromolecular Symposia, Vol. 216, No. 1, 2004, pp. 293-299. doi:10.1002/masy.200451227

[26] Z. Q. Liu, A. M. Cunha, X. S. Yi and C. A. Bernardo, "Thermal characterizations of wood flour/starch cellulose acetate compounds," Journal of Macromolecular Science Part B Physics, Vol. 40, No. 3-4, 2001, pp. 529-538. doi:10.1081/MB-100106175

[27] D. S. Rosa, C. G. F. Guedes, F. Casarin and F. C. Bragança, "The Effect of the Mw of PEG in PCL/CA Blends,” Polymer Testing, Vol. 24, No. 5, 2005, pp. 542548. doi:10.1016/j.polymertesting.2005.02.002 